\title{
Microbes, magnetism, and microscopy
}

\author{
E. Dan Dahlberg ${ }^{\text {a }}$, R.B. Proksch ${ }^{a}$, B.M. Moskowitz ${ }^{\text {b }}$, D.A. Bazylinski ${ }^{\text {, }}$, \\ R.B. Frankel ${ }^{\mathrm{d}}$ \\ ${ }^{a}$ School of Physics and Astronomy, University of Minnesota, Mpls., MN 55455, USA \\ ${ }^{\mathrm{b}}$ Institute for Rock Magnetism, University of Minnesota, Mpls., MN 55455, USA \\ ${ }^{\mathrm{c}}$ Marine Science Center, Northeastern Uniwersity, East Point, Nahant, MA 01908, USA \\ ${ }^{d}$ Dept. of Physics, Cal Poly State University, San Luis Obispo, CA 93407, USA
}

\begin{abstract}
An accurate quantification of magnetic force microscope images has been accomplished. The magnetosomes produced by magnetotactic bacteria, an ideal micromagnetic model system, were the specimens used for the quantification (a moment on the order of $10^{-13} \mathrm{emu}$ ).
\end{abstract}

Magnetic force microscopes (MFMs) are a variant of the non-contact AFM in which magnetic fields have been imaged with submicron resolution. These instruments consist of a small magnetic probe, typically on the end of a vibrating cantilever, which is scanned above a magnetic sample. The interactions of the magnetic probe with the stray micromagnetic fields from the sample are mapped to form an image that yields information about the micromagnetic structure of the sample. Although imaging is straightforward, quantification of the images, i.e., a determination of the magnetization of the specimen under investigation, has been difficult. The main difficulties are a lack of well characterized tips, a well defined, simple magnetic moment to investigate, and the invasive nature of the measurements [1], i.e., the fields of the tip can alter the specimen magnetization and vice versa. An added complication in the MFM is sensitivity to the second derivative with respect to distance of the sample generated magnetic fields. In recent work [1] to be summarized here, we were successful in circumventing these difficulties and successfully quantifying MFM images of the magnetic field produced by a chain of magnetosomes in a magnetotactic bacterium.

Magnetotactic bacteria (MTB) orient and migrate along the geomagnetic field towards favorable habitats, a behavior known as magnetotaxis [2]. MTB produce intracellular chains of permanent single magnetic domain particles of magnetite $\left(\mathrm{Fe}_{3} \mathrm{O}_{4}\right)$, or gregite $\left(\mathrm{Fe}_{3} \mathrm{~S}_{4}\right)$. The mineral particles and their enveloping membrane, called magneto- somes, are characterized by a narrow size distribution, specific crystallographic orientations, and species-specific crystal morphologies.

Magnetosomes are usually organized in one or more linear chains, with the crystallographic magnetic easy axes $\left(\langle 111\rangle\right.$ for $\mathrm{Fe}_{3} \mathrm{O}_{4}$ ) of each particle also aligned along the chain axis. The size specificity and crystallographic orientation of the chain assembly is optimally designed for magnetotaxis [3]. The magnetic dipole moments of individual magnetotactic bacteria have previously been inferred by a variety of techniques to be in the range of $10^{-13}$ to $10^{-12}$ emu.

Interest in the biomineralization of magnetite by bacteria has initiated research in several different fields including microbiology, physics, and paleomagnetism. For paleomagnetism, biogenic magnetic minerals can be deposited in sediments and preserve a record of the ancient geomagnetic field [4]. For physics, biogenic magnetic minerals provide a novel source of single domain particles for experimental studies in fine particle magnetism [5]. In our work, we used the magnetosome chains as very simple magnetic dipole moments for quantification of the images obtained by MFM. An added bonus of this work is a measurement of the moment of the bacteria which provides a detailed characterization of the chains and represents a measurement of a single moment of on the order of $10^{-13}$ emu.

In what follows, we will briefly discuss the experimental techniques, the background necessary for understanding 
the MFM imaging data, and detail the model used for calculating the moment of the magnetosome chains from the MFM images.

Freeze-dried cells of the magnetotactic bacterial strain MV1 were used in our study [6]. Individual magnetite particles in MV1 are truncated hexahedral prisms with average dimensions of $53 \times 35 \times 35 \mathrm{~nm}$. Particles are arranged in a single linear chain with an average number of magnetosomes / cell of 10 [7]. The freeze-dried cells had a bulk coercivity of 385 Oe at room temperature. Specimens for the MFM study were prepared by rehydrating freeze-dried cells, and allowing them to dry on a glass slide [8] prior to AFM and MFM studies.

For MFM imaging, the tip is scanned over the surface such that only long range forces, e.g., the magnetic forces, are acting on the cantilever. To separate the long range or magnetic forces from the short range topographic forces, several non-contact MFM images at different interaction strengths (or equivalently, different tip-sample separations) were made. Analysis of these scans allowed separation of the topographic and magnetic forces.

The MFM image of three bacteria showing a clear magnetic image is shown in Fig. 1a. This image, taken with a high coercivity tip, shows both attractive and repulsive magnetic interactions as expected. In the situation when imaging with magnetically soft tips, the fields from the magnetosomes can affect the image. For example, shown in Fig. 2 is an MFM image made with a a low coercivity magnetic tip which resulted in the interaction between the tip and dipolar field from the cell always being attractive.

Most models of ac mode MFM assume that the cantilever acts as a simple harmonic oscillator. Magnetic interactions enter in as perturbations, shifting the equilibrium position of the cantilever up or down and shifting the resonant frequency up or down by an amount proportional to $F_{z}^{\prime}$, the $z$ component of the force gradient acting between the tip and sample [9]. In analyzing the behavior
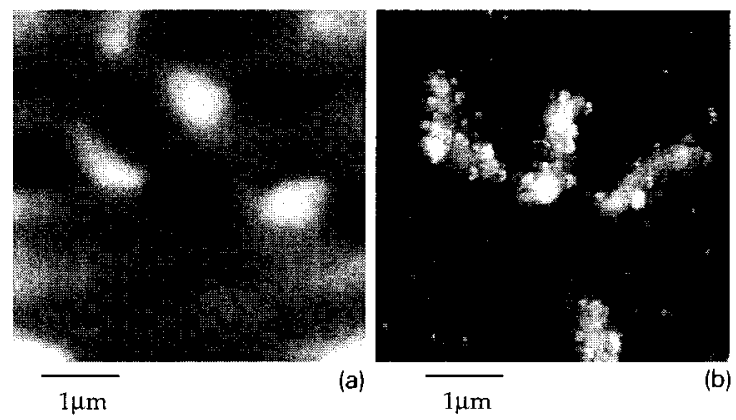

Fig. 1. (a) MFM image of freeze dried MV-1 bacteria. The magnetic tip is located approximately $100 \mathrm{~nm}$ from the magnetosome chain. The light and dark regions correspond to the two poles of the magnetic moment. (b) A contact image of the same freeze-dried cell shown in (a).

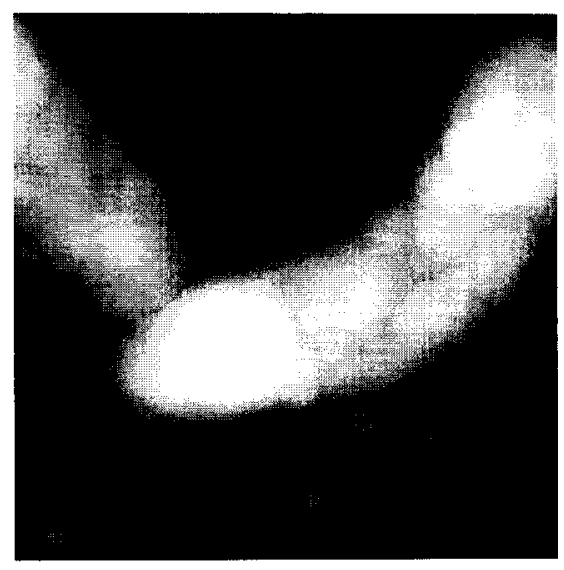

Fig. 2. An MFM image of a bacterium made with a low coercivity tip. Because the coercivity is less than the fields from the magnetosomes, the MFM tip is always aligned with the magnetosome field and thus is always attracted to the sample.

of an MFM, we make the following assumptions: (1) the magnetic perturbations are small and can be treated as constant over the range of the cantilever's motion, (2) the cantilever can be modeled as a driven simple harmonic oscillator with damping, described by a simple Lorentzian, and (3) the cantilever is being driven at a particular frequency off resonance which results in the maximum amplitude change for a resonance shift. In this case, the amplitude changes of the vibrating cantilever will be given by [2]:

$\Delta A=\frac{\partial A}{\partial \omega_{\mathrm{D}}} \mid \omega_{\mathrm{D}}^{\max } \frac{F_{z}^{\prime}}{2 k}$,

where $k$ is the spring constant of the cantilever and $\partial A /\left.\partial \omega_{\mathrm{D}}\right|_{\omega_{\mathrm{D}}^{\max }}$ is the slope of the amplitude response curve at the cantilever drive frequency, $\omega_{\mathrm{D}}$, which can be experimentally determined.

For the case where the cantilever is constrained to oscillate along the $z$ axis, the force gradient acting on a magnetic tip is given by [10]

$F_{z}^{\prime}=\int_{\text {Tip Volume }} \boldsymbol{m}\left(\boldsymbol{r}^{\prime}\right) \cdot \frac{\partial^{2} \boldsymbol{B}}{\partial z^{2}}\left(\boldsymbol{r}-\boldsymbol{r}^{\prime}\right) \mathrm{d}^{3} \boldsymbol{r}^{\prime}$.

The response of an MFM depends on a number of parameters which must be determined for image quantification. As a starting point for the magnetic parameters of the thin film coating on the tip, we measure the bulk properties of a magnetic film co-sputtered with the cantilevers. These values are then used as the starting parameters in fitting the image of a well characterized magnetic system, e.g., bits in a thin film magnetic hard disk [1].

Another important paramenter is the tip-sample separation distance. It was left as a nonlinear fitting parameter constrained to be between 50 and $200 \mathrm{~nm}$. The typical value was $65 \mathrm{~nm}$. It was found that changing this value 


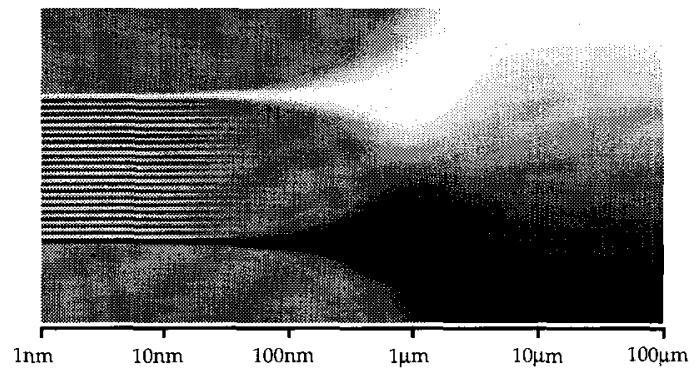

Fig. 3. The calculated field gradients from the magnetosome chain model, along the chain, as a function of height, $z$, above the bacterium and lateral position, $x$. The grayscale contrast has been normalized to the maximum absolute value of the field for each value of $z$. Note that the relative maxima and minima for heights up to $z=1.5 \mu \mathrm{m}$ are directly above the ends of the chain.

had a large effect on the fitted moment; it is probably the largest source of error in the fit. The limits of this value were determined by the AFM images (see Fig. 1b) which indicated the total cell thickness was on the order of 100 nm.

For fitting the MFM data of the imaged magnetosomes to their moment, it was necessary to determine the field derivatives of the magnetic field arising from the magnetosomes. Based on TEM studies of MV-1, which indicate that the magnetosomes are roughly truncated hexahedral prisms, we modeled an individual magnetosome as a uniformly magnetized cylinder of length $53 \mathrm{~nm}$ and a diameter of $35 \mathrm{~nm}$. We chose the cylindrical geometry even though it ignores the edge effects probably present in real magnetosomes [11]. As determined by the TEM studies, the magnetosomes were separated by ca. $10 \mathrm{~nm}$. Using these as the model parameters, the relevant magnetic field gradients can be calculated. An example of the calculated gradients are shown in Fig. 3.

It is now possible to make an estimate of the moment of the bacteria from Fig. 1. Close inspection of Fig. 3 shows that the $z$ component of the field derivative is a maximum or minimum directly over the ends of the chain of magnetosomes until a height of roughly $1.5 \mu \mathrm{m}$. This implies that as long as the MFM responds to the portion of the field below the $z=1.5 \mu \mathrm{m}$, the distance between the maxima and minima of the MFM response should correspond to the total length of the chain of magnetosomes. Using this argument, we obtained a moment value of $6 \times 10^{-13} \mathrm{emu}$.

Next, using the above estimate as a starting point, a nonlinear fitting routine was employed to further refine the measurement of the magnetic moment from the nanoscale magnetic assembly. The details of this fitting are presented elsewhere [1]; however, the fitting technique involved minimization of a 'merit of fit' function. This full fitting process resulted in a magnetic moment for a bacterium on the order of $1 \times 10^{-13} \mathrm{emu}$, which represents the smallest quantitative measurement of a magnetic moment to date.

By using well defined and simple magnetic specimens, it is possible to quantify MFM images. This first step provides a path to quantify MFM images on a routine basis. The quantification process has provided a measurement of the magnetic moment in magnetotactic bacterium. This moment measurement of $4+4 /-3.5 \times 10^{-13} \mathrm{emu}$ represents a moment approximately 6 orders of magnitude smaller than can be measured by standard techniques.

Acknowledgements: The MMC gratefully acknowledge support of this research by the ONR. The IRM acknowledges support from the NSF and the Keck Foundations. R.B.F. and D.A.B. were supported by the ONR.

\section{References}

[1] R.B. Proksch, E. Dan Dahlberg, B.M. Moskowitz, D.A. Bazylinski and R.A. Frankel, submitted.

[2] S. Mann and R.B. Frankel, in Biomineralization: Chemical and Biochemical Perspectives, S. Mann, J. Webb and R.J.P. Williams, eds., pp. 389-426 (VCH, New York, 1989).

[3] D.A. Bazylinski, A.J. Garratt-Reed and R.B. Frankel, Microscopy Res. Techn. 27 (1994) 389.

[4] B.M. Moskowitz, R.B. Frankel and D.A. Bazylinski, Earth Planet. Sci. Lett. 120 (1993) 283.

[5] R. Proksch and B. Moskowitz, J. Appl. Phys. 75 (1994) 5894.

[6] D.A. Bazylinski, in Iron Biominerals, R.B. Frankel and R.P. Blakemore, eds., pp. 69-77 (Plenum, New York, 1990).

[7] N.H.C. Sparks, S. Mann, D.A. Bazylinski, D.R. Lovley, H.W. Jannasch and R.B. Frankel, Earth Planet. Sci. Lett. 98 (1990) 14.

[8] When the whole cells were imaged, it was impossible to identify a clear magnetic signal. This lack of observation of a magnetic moment was due to the small magnitude of the moment and because the magnetsomes are deep within the cell body and the MFM tip was constrained to remain a distance on the order of $0.5 \mu \mathrm{m}$ from the internal magnetosomes. The freeze drying reduced the bulk of the cell tissue and brought the magnetosomes closer to the cell surface.

[9] D. Rugar, H.J. Mamin, P. Guethner, S.E. Lambert, J.E. Stern, I. McFadyen and T. Yogi, J. Appl. Phys. 68 (1990) 1169.

[10] P. Grütter, D. Rugar and H.J. Mamin, Ultramicroscopy 47 (1992) 393.

[11] In practice, the variation in the calculated magnetic field between a cylindrical and cubical geometry at realistic tipsample separations is quite small. 